\title{
puesto central de defensa pasiva
}

\author{
R. EVAN KENNEDY, ingeniero
}

La ciudad de Portland, Oregón (EE. UU.), está terminando la construcción de un centro subterráneo, destinado a la defensa civil del casco urbano, que, en caso de emergencia, tendrá por objeto la dirección y rehabilitación de los servicios que un ataque o desastre local pudieran devastar. El refugio dispondrá de facilidades para poder acomodar al gobierno con sede en la ciudad. Este proyecto, de larga incubación hasta llegar al estado de ejecución, ha previsto la instalación en el refugio de los servicios siguientes: policía, contra incendios, ingeniería, médicos, beneficencia y jefaturas varias. También se han previsto los servicios de prensa y radio.

El refugio podrá alojar a unas 300 personas por espacio de siete días consecutivos, sin que sea preciso salga nadie de su interior; su estructura, en forma de bóveda cilíndrica de hormigón, tiene un espesor de más de $0,60 \mathrm{~m}, 26 \mathrm{~m}$ de luz y $42,50 \mathrm{~m}$ de longitud. Parte de esta longitud ha sido utilizada para la construcción de una segunda planta de entramado metálico y forjado de hormigón. El resto de la longitud, es decir, los otros $16,50 \mathrm{~m}$ constituyen un espacio suficiente para la sala de operaciones, cuyos muros laterales y la parte posterior del que cierra el refugio servirán para la colocación de mapas.

Además de todas estas dependencias, la entrada estará formada por una estructura para la instalación de la sala de ventiladores, servicios de descontaminación y paso de identificación de las personas que pretendan entrar en el refugio.

\section{Características de la estructura}

La cubierta inmediata de la estructura está constituída por un segmento cilíndrico, atirantado, de unos $14 \mathrm{~m}$ de radio y $8,50 \mathrm{~m}$ de flecha, con un espesor uniforme de $0,66 \mathrm{~m}$ en toda su longitud. La razón de haber preferido el espesor uniforme a un espesor variable entre clave $y$ arranques, o a un sistema de lámina nervada, ha sido que el terreno que debía cubrir la bóveda, de $2.080 \mathrm{~kg} / \mathrm{m}^{\mathrm{s}}$ de peso, daba una carga sobre la cubierta de unos $3.200 \mathrm{~kg} / \mathrm{m}^{2}$, lo que ocasionaba grandes momentos en la clave. De haber elegido la solución nervada, los nervios se tenían que aproximar mucho, y, como se necesitaría la misma cantidad de acero en la clave para resistir dichos momentos en cualquier solución, se descartó el principio en apariencia económico, del espesor variable por el espesor uniforme adoptado, que, además, introducía una gran economía de encofrados y presentaba grandes ventajas y seguridades contra la penetración de radiaciones y de explosiones directas contra el hormigón.

La lámina se ha reforzado con dos capas de armaduras transversales, con barras espaciadas a $18 \mathrm{~cm}$. Estas armaduras disponen de estribos espaciados a $46 \mathrm{~cm}$. Longitudinalmente se ha reforzado con barras espaciadas a $0,30 \mathrm{~m}$. Los empalmes de estas armaduras se han solapado $0,60 \mathrm{~m}$, dejando un espacio, entre ellos, equi-

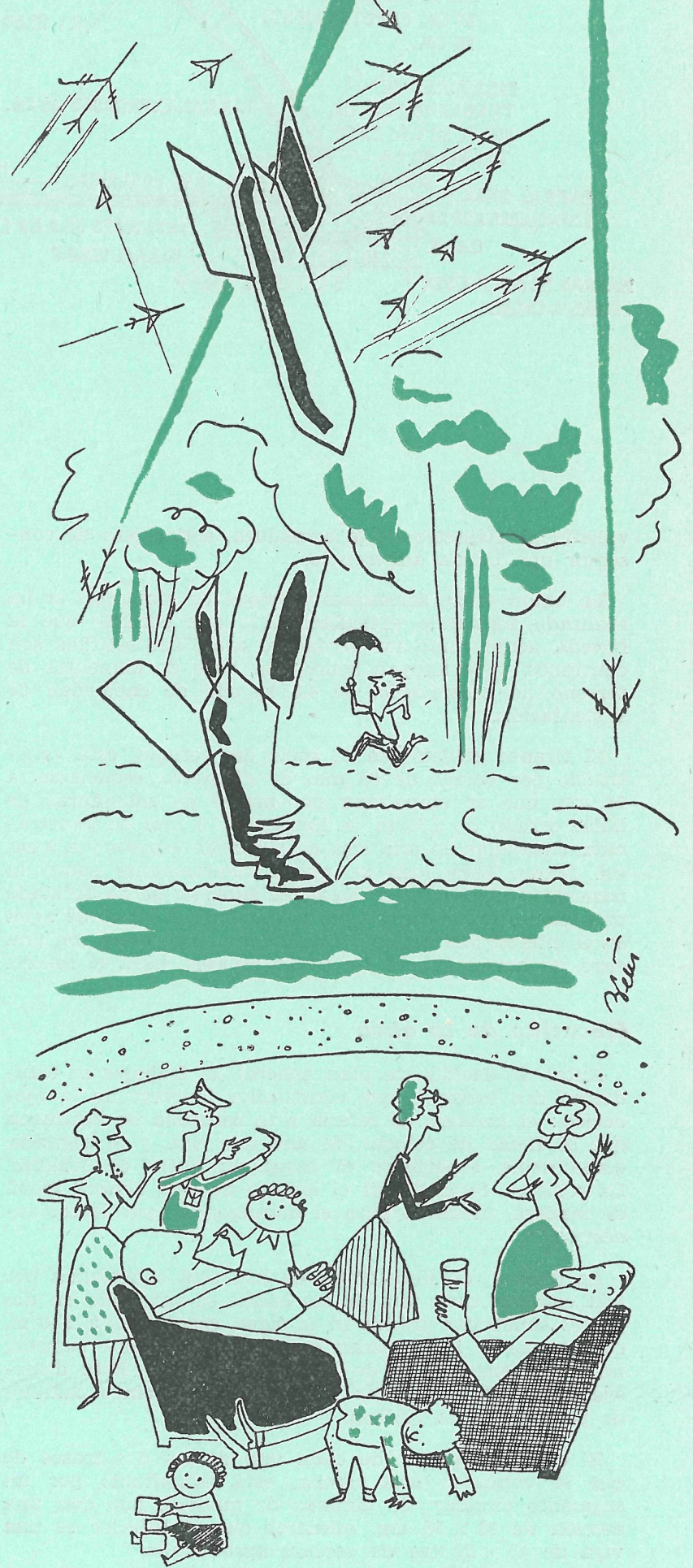

INSTITUTO TECNICO DE LA CONSTRUCCION Y DEL CEMENTO 


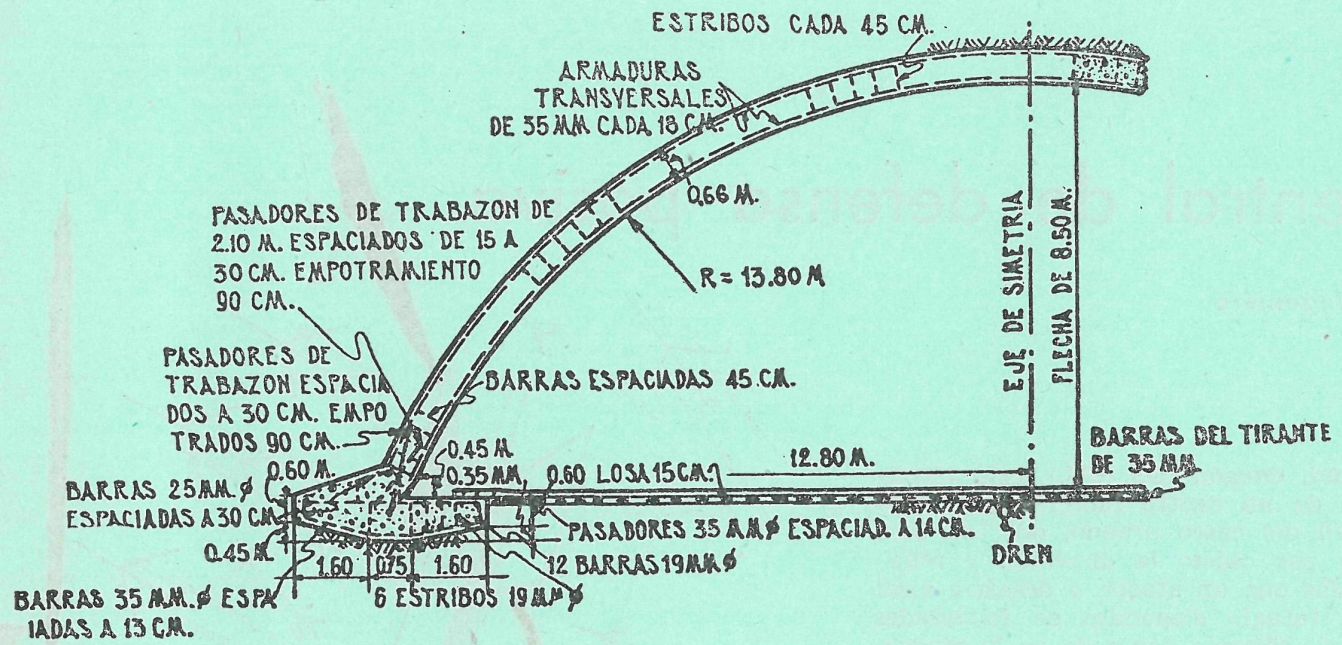

sección

Semialzado del refu. gio, mostrando la disposición de armadu. as.

valente al diámetro de la armadura, con objeto de conseguir una buena adherencia.

La unión entre arranques y bloque de cimientos se ha realizado formando articulación, lo que permite que la bóveda actúe como un verdadero arco. Los estribos son continuos, y se apoyan sobre una base de cimientos de terreno no perturbado, de $4,8 \mathrm{~kg} / \mathrm{cm}^{2}$ de capacidad de sustentación.

El tirante, situado en el suelo del refugio, está constituído por barras de $35 \mathrm{~mm}$ de diámetro, espaciadas a $14 \mathrm{~cm}$, que se empalman por medio de soldaduras de doble cordón de $0,30 \mathrm{~m}$ de longitud y $8 \mathrm{~mm}$ de penetración, encajadas en una losa de $15 \mathrm{~cm}$ de espesor y a $9 \mathrm{~cm}$ del fondo. Esta losa se apoya directamente sobre la base de cimientos, y en su parte superior se ha formado una membrana que evita pueda penetrar el vapor de agua en el interior del refugio; finalmente, se recubrió con una capa de hormigón, de $10 \mathrm{~cm}$ de espesor, para el solado.

\section{Ejecución de la obra}

Antes de decidir un plan general de trabajos se estudiaron dos factores, que constituían la clave de la ejecución: el primero se refería a la cantidad de hormigón que se había de colocar de una sola vez, y el segundo, cómo debía soportarse el hormigón antes de fraguar. La solución consistió en el empleo de una cimbra móvil de madera, utilizada para el hormigonado por trozos sucesivos.

La cimbra, de unos $26 \mathrm{~m}$ de luz, está constituída por tres cerchas, cada una de las cuales se subdivide en dos partes simétricas unidas en la clave, espaciadas a $2,40 \mathrm{~m}$, con lo que se logra poder hormigonar, sin interrupción, un trozo longitudinal de bóveda de 7,15 m. Esta disposición permitió terminar la cubierta con cinco cambios de posición de la cimbra.

El cordón superior de cada uno de los dos trozos de que se compone una cercha, está constituído por un segmento circular de láminas de madera, que dan una sección de $13 \times 35 \mathrm{~cm}$, mientras que el inferior es una viga de $15 \times 20 \mathrm{~cm}$ de sección aproximada.
El ciclo de trabajo de cada uno de los trozos de bóveda, que absorbe $155 \mathrm{~m}^{3}$ de hormigón, ha sido de quince días. La operación de hormigonado propiamente dicha requería tan sólo seis horas; el resto se dedicaba al cambio de posición de la cimbra, colocación de armaduras y fraguado suficiente hasta lograr la resistencia conveniente para llegar a la autosustentación del trozo terminado.

El hormigón se vertió de tal forma que se iba equilibrando a medida que avanzaba la operación, vertiendo a uno y otro lado de la bóveda. Antes de desencofrar se curaba el hormigón hasta conseguir una resistencia de $140 \mathrm{~kg} / \mathrm{cm}^{2}$ a los cuatro días. La resistencia final prevista, a los veintiocho días, era de $210 \mathrm{~kg} / \mathrm{cm}^{2}$. El desencofrado se realizó con la ayuda de cajas de arena y gatos hidráulicos, utilizando unos carriles que se habian colocado, a cada lado de la nave, para la traslación de la cimbra de una posición determinada a la nueva, correspondiente al trozo que se debía hormigonar a $7,15 \mathrm{~m}$ de distancia, y asi sucesivamente.

El fondo de la cimbra se recubrió con una capa de material aislante acústicamente, de $38 \mathrm{~mm}$ de espesor, que forma el techo de la nave. La colocación de este material sobre el encofrado necesitó día y medio por trozo. Además de los buenos resultados obtenidos con este material, bajo su aspecto acústico, permitió el reempleo sucesivo del fondo de la cimbra, sin necesidad de reparación alguna del encofrado, a pesar de los cambios sucesivos de posición.

Aunque la losa de la solera se hormigonó independientemente, era necesario la posición conveniente de la parte correspondiente al tirante en el momento de desencofrar cada uno de los trozos.

La obra no ha sido recubierta de tierra todavía, pero se han colocado extensómetros en los lugares donde se espera aparezcan las mayores solicitaciones, con objeto de poder controlar los esfuerzos en el momento de rellenar el hueco. El espesor mínimo de tierra que gravitará sobre la bóveda es de unos $1,50 \mathrm{~m}$, y, debido a la consideración de esta sobrecarga, la tierra se vaciará tomando las debidas precauciones para distribuir su peso lo más inofensivamente posible. 


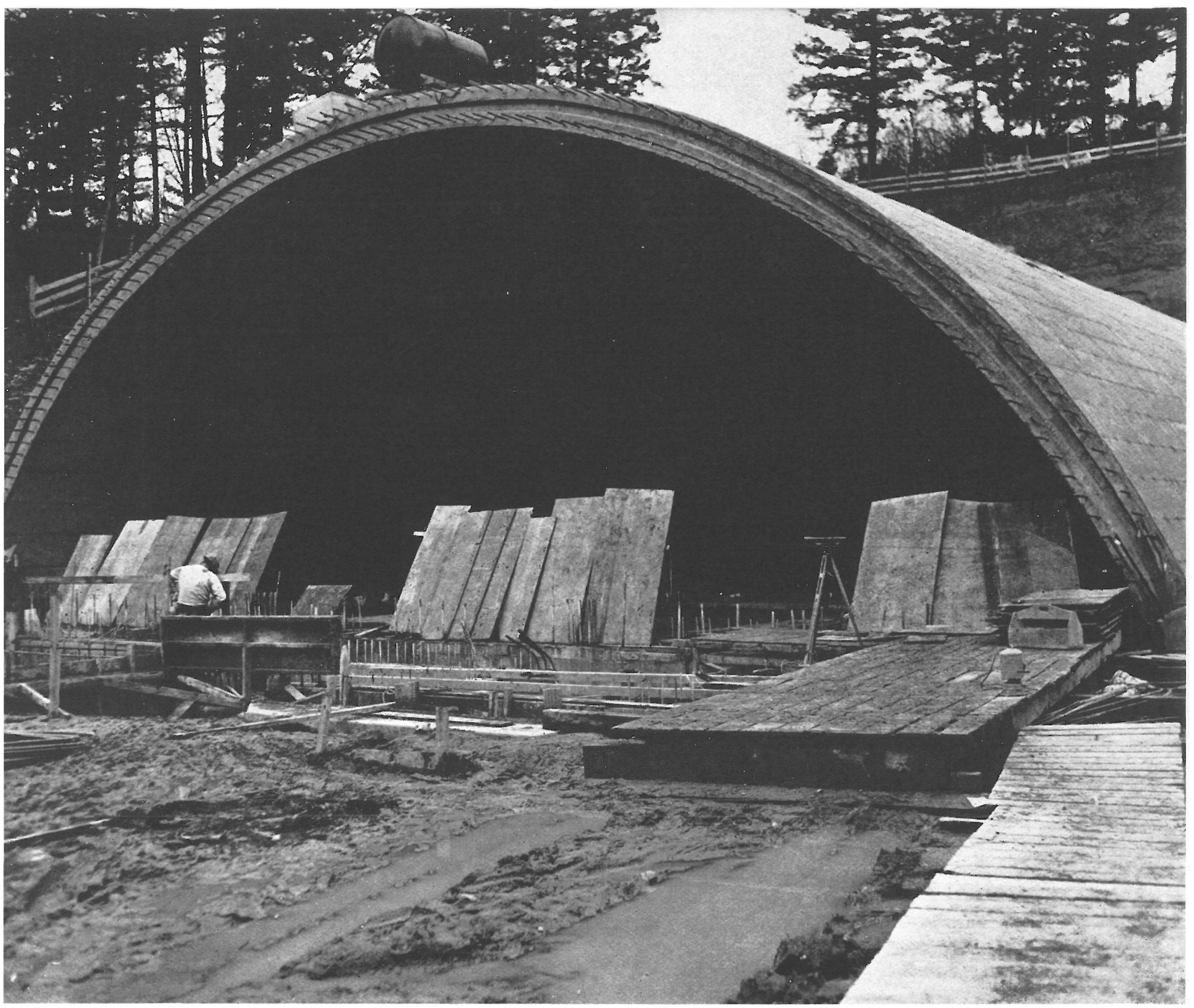

Una fase de la construcción del refugio.

\section{Instalaciones}

La toma de aire para la ventilación del refugio se verifica por medio de una chimenea, que sale al exterior y conduce el aire a la sala de ventiladores, impulsándolo al interior. Si el aire no está contaminado, pasa por filtros y serpentines de refrigeración o calefacción, y, si está contaminado, se le filtra mediante un dispositivo especial para eliminar las partículas radiactivas que pudiera contener.

Si la contaminación del aire exterior fuera tan grande que no se pudiese utilizar para la ventilación, se cierra el circuito en el interior del refugio, se hace pasar el aire por filtros de carbón activo, se le añade oxígeno, se le enfría y se le distribuye por el interior del refugio si- guiendo ciclos sucesivos. Algunas veces se podrá añadir una pequeña cantidad del aire exterior, previamente purificado para mejorar la calidad del aire empleado en el circuito cerrado.

El interior del refugio se halla a presión, y, por tanto, no cabe temer a penetraciones del aire del exterior. Las puertas se han dispuesto en tal forma, que se pueden abrir o cerrar permaneciendo el aire a presión. A fines de ventilación, el edificio se ha dividido en dos zonas: una normal y otra de compensación en los casos de emergencia. Cuando la ventilación ha de subvenir a las necesidades de 300 personas, la carga de la instalación es considerable. El cambio de circulación de aire de una a otra zona, se efectúa por una serie de válvulas y ventiladores capaces de orientar convenientemente la corriente. 

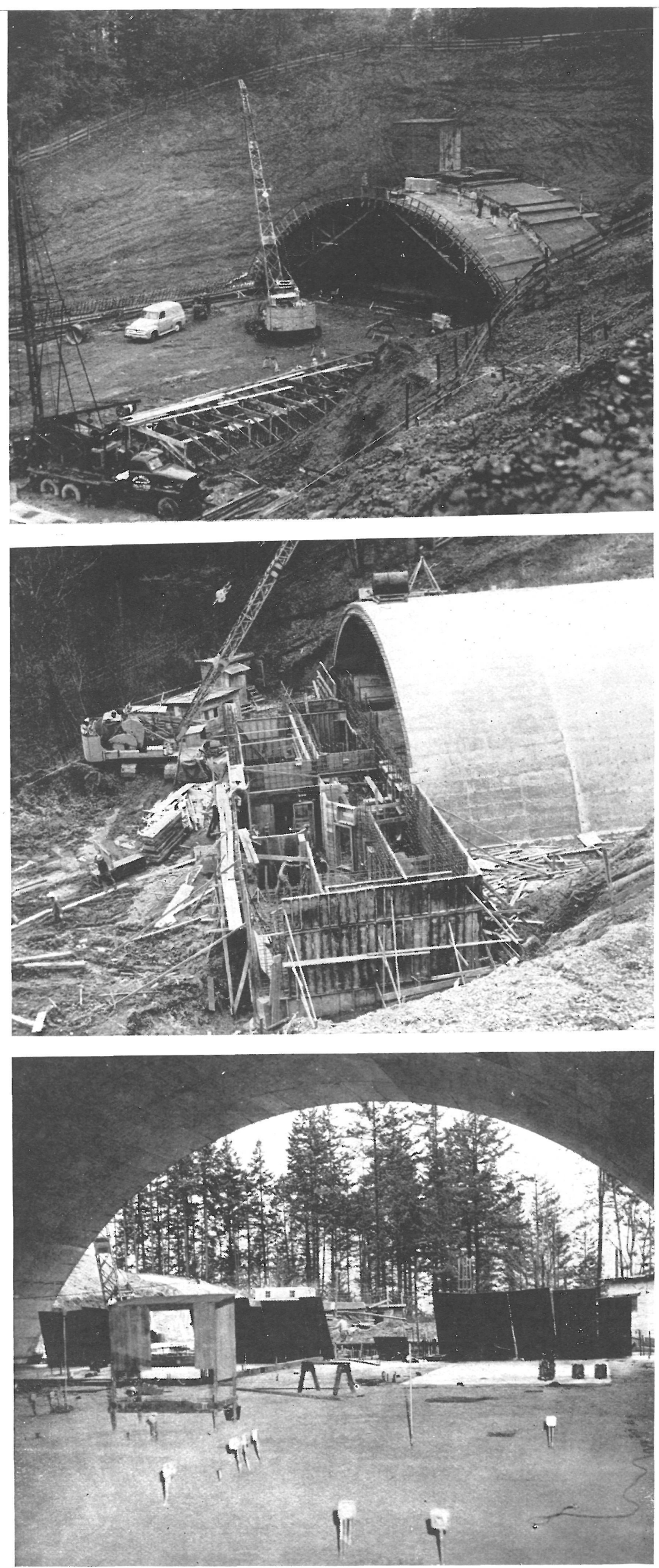

Los materiales de construcción se llevaban, por medio de una grúa móvil, refugio.

Construcción de los servicios y entrada de la parte de fachada del refugio.

Vista del refugio-antes de cerrarlodesde el interior.

Como el edificio es subterráneo, los cambios de temperatura no tienen gran importancia, y, por ello, las pérdidas de calor son relativamente de menor consideración. Esto no obstante, habrá momentos en que se necesite la calefacción; a este objeto, un grupo electrógeno de $90 \mathrm{kw}$ suministrará el $75 \%$ de estas necesidades, que, de no ser suficiente, un disparador automático hará entrar en acción un segundo generador de calor que aprovecha el recuperado del sistema de refrigeración. Cuando la temperatura aumenta excesivamente, un termostato aisla, automáticamente, al segundo generador.

Todos los servicios operan eléctricamente $y$, por tanto, la instalación dispone de medios propios generadores. Cuando la carga de la instalación rebasa ciertos límites, el grupo generador entra en servicio automáticamente; en caso contrario, y también automáticamente, se aisla, dejando a las líneas exteriores que alimenten el sistema.

El refugio dispondrá de una instalación de células fotoeléctricas para la identificación del personal que entra en el edificio y, para ello, toda persona que entre ha de pasar por un lugar donde existe un aparato emisor de televisión, puesto en marcha por la célula fotoeléctrica, que proyecta en el interior su imagen; una vez ante la puerta, presenta su carta de identidad ante una pantalla que la retransmite al interior $y$, finalmente, se le abre o deja cerrada, automáticamente, la puerta de acceso.

Para conservar un ambiente agradable en el interior del edificio subterráneo, se ha previsto la toma de un gran volumen de aire, un color de tonos adecuados y pantallas de rayos ultravioleta para refrescar el aire. Estas consideraciones y las precauciones tomadas contra la penetración de humedad, harán un lugar acogedor para el trabajo en invierno y verano.

Para las comunicaciones normales se han previsto líneas telefónicas, pero, en caso de emergencia, el refugio se comunica con el exterior por medio de aparatos de radio.

J. J.U. 